\title{
Updated Validation and Deposition Tools in the Phenix GUI
}

\author{
Billy K. Poon ${ }^{a}$, Pavel V. Afonine ${ }^{a, b}$, Dorothee Liebschner ${ }^{a}$, Nigel W. Moriarty ${ }^{a}$, \\ Oleg V. Sobolev ${ }^{\mathrm{a}}$, and Paul D. Adams ${ }^{\mathrm{a}, \mathrm{c}}$
}

a) Molecular Biophysics and Integrated Bioimaging, Lawrence Berkeley National Laboratory, Berkeley, CA, USA, bkpoon@/bl.gov, pafonine@lbl.gov, dcliebscher@/b.gov,nwmoriarty@/bl.gov,osobolev@/bl.gov,pdadams@/bl.gov b) Department of Physics, Shanghai University, Shanghai, People's Republic of China

c) Department of Bioengineering, University of California Berkeley, Berkeley, CA, USA

The Phenix (Python-based Hierarchical Environment for Integrated Xtallography) software package is a comprehensive collection of tools for determining macromolecular structures. Traditionally, the software used reciprocal-space data (e.g. X-ray/neutron crystallography) for structure determination, but with recent advances in cryo-electron microscopy (cryo-EM) providing higher resolution electron density maps, the software can be applied to real-space data as well. Previous updates to the graphical user interface for Phenix include functionality for validating cryo-EM maps and models, as well as a new unified interface for these validation tools.

With the most recent builds of Phenix, we applied this approach to the $\mathrm{X}$-ray/neutron crystallography validation tools and added a new tool for preparing a model file in mmCIF format for deposition into the Protein Data Bank (PDB). The result is a consistent validation interface for both X-ray/neutron crystallography and cryo-EM structures that can export a table of statistics for publication (e.g. Table 1) and export an mmCIF model file suitable for deposition into the PDB. 